USTC-ICTS-13-02

\title{
Modulating the phase structure of black D6 branes in canonical ensemble
}

\author{
J. X. Lu1 and Ran Wei2 \\ Interdisciplinary Center for Theoretical Study \\ University of Science and Technology of China, Hefei, Anhui 230026, China
}

\begin{abstract}
There exists a dramatic difference in phase structure between charged black Dp-branes with $p<5$ and charged black D5 or D6 branes. In [1, we found that the phase structure of charged black D5 system can be changed qualitatively to that of charged black Dp with $p<5$ by adding the delocalized D1 branes but the similar change does not happen for the charged black D6 system through adding the delocalized D2 branes (Note that both D1/D5 and D2/D6 belong to the same type of $\mathrm{D}(\mathrm{p}-4) / \mathrm{Dp}$ system $)$. Adding the delocalized D4 branes to the black D6 branes doesn't work, either. In this paper, we consider adding the delocalized D0 branes, the only remaining lower dimensional branes, to the black D6 system for this purpose. We find that the delocalized charged black D0-branes alone share the same phase structure as the charged black D6 branes, having no van der Waals-Maxwell liquid-gas type. However, when the two are combined to form D0/D6, the resulting phase diagram finally gets changed dramatically to the wanted one, containing now the above liquid-gas type. This change arises from the interaction between D6 and the delocalized D0.
\end{abstract}

\footnotetext{
${ }^{1}$ E-mail: jxlu@ustc.edu.cn

${ }^{2}$ E-mail: wei88ran@mail.ustc.edu.cn
} 


\section{Introduction}

Understanding the nature of black hole thermodynamics may teach us lessons about quantum gravity. The underlying phase structure, in particular its possible universality and the associated phase transitions, can be useful in this regard. For example, if the phase structure is universal for black holes with different asymptotic spacetimes (including AdS one), this may hint us that there exits a general holography for these spacetimes [2, 3] (not just true only for AdS case). Motivated by this, it has been recently shown [2, 3] that a large part of the phase structure of a black hole in asymptotically anti-de Sitter (AdS) space [4, 5, 6] is not unique to the AdS black hole, but actually shared universally by suitably stabilized black holes, say, in asymptotically flat space, even in the presence of a charge $q$. Concretely, a chargeless (suitably stabilized) black hole in asymptotically flat space can also undergo a Hawking-Page transition at certain temperature, now evaporating into a regular "hot flat space" instead of a regular "hot empty AdS space" as for an AdS black hole. Moreover, when $q \neq 0$, there exists also a critical charge $q_{c}$ and for $q<q_{c}$, the phase diagram universally contains a van der Waals-Maxwell liquid-gas type phase structure along with a line of first-order phase transition terminating at a second-order critical point with a universal critical exponent for specific heat as $2 / 3$.

An isolated asymptotically flat black hole, unlike the AdS one, is thermodynamically unstable due to its Hawking radiation and needs to be stabilized first before its phase structure can be analyzed properly. The standard practice for this is to place such a system inside a finite spherical cavity [7] with its surface temperature fixed. So a thermodynamical ensemble is considered which can be either canonical or grand canonical, depending on whether the charge inside the cavity or the potential at the surface of the cavity is fixed [8]. We focus in this paper on the canonical ensemble, i.e., the charge inside the cavity is fixed.

With the advent of AdS/CFT correspondence, the Hawking-Page transition for AdS black hole 'evaporating' into regular "hot empty AdS space" at certain temperature [4] corresponds to the confinement-deconfinement phase transition in large $N$ gauge theory [9]. The charged AdS black hole has a phase structure of van der Waals-Maxwell liquid-

gas type, giving rise to the so-called catastrophic holography as discussed in [5]. As mentioned above, this kind of phase structure of AdS black hole is actually universal and is the result of the boundary condition rather than the exact details of asymptotical metrics which can be either flat, $\operatorname{AdS}$ or dS [2, 3]. The boundary condition realized in each case by the reflecting wall provides actually a confinement to the underlying system. This may suggest that the AdS holography is a result of such confinement rather than 
the detail properties of AdS space. Then the natural speculation as mentioned earlier is that a similar holography should hold even in asymptotically flat space. If such a holography holds indeed, the natural and interesting questions are: how do we define the corresponding field theory on the underlying holographic screen 3 which is supposed to be the spherical cavity and what do the various thermal dynamical phase transitions correspond to in the field theory so defined?

We have recently found [10, 11] that the same characteristic phase structure (including a Hawking-Page transition) of stabilized uncharged (Schwarzschild) black holes was also shared by the stabilized uncharged black p-branes in D-dimensional asymptotically flat spacetime (with the brane worldvolume dimensions $d=1+p$ ) in string/M theory. However, in the charged case, the van der Waals-Maxwell liquid-gas type phase structure mentioned earlier, with its characteristic behavion 4 shown in Fig. 1, holds true only for the stabilized charged black p-branes with the corresponding $\tilde{d} \equiv D-d-2>2$ (note that $1 \leq \tilde{d} \leq 7$, where $\tilde{d}+2$ is the number of the spatial dimensions transverse to the brane). For $\tilde{d}=1(<2)$, the underlying phase structure is qualitative different and actually resembles that of chargeless case instead. For the $\tilde{d}=2$ case, there exists a 'critical charge' $q_{c}=1 / 3$ and we have three subcases to consider, depending on the charge $q>q_{c}, q=q_{c}$

\footnotetext{
${ }^{3}$ For an asymptotically flat black hole without an origin from branes in string/M theory, establishing such a field theory description will be extremely difficult, not mentioning the issue associated with the cavity. For asymptotically flat black branes, to be discussed next, it is very natural to suppose that there exists an associated dual field theory arising on the worldvolume of the corresponding branes. Here the issue is how to consider properly the cavity effect, which may be viewed as imposing certain boundary conditions on the fields. Note that such a field theory, if exists at all, is neither supersymmetric nor conformal in general, due to the presence of cavity. Pursuing this dual field theory and the corresponding holography will be our future effort and what has been discussed for the AdS cases given in [5, 6] should be a good guidance. Our purpose here and the near-future efforts will be on the understanding of the potential universal phase structure and its correlation with the underlying interaction from the gravity side for branes in string/M theory.

${ }^{4}$ The so-called reduced quantities $b_{q}(x), \bar{b}, x, q, q_{c}$ in Fig. 1 are defined in [10] and will also be given later in section 3 for D6-branes. $b_{q}(x)$ is the reduced inverse of local temperature of charged black Dp branes under consideration at the surface of cavity. $\bar{b}$ is the reduced inverse of the given temperature of cavity. $x$ is the reduced horizon of the black system and $q$ is its reduced charge with its corresponding critical $q_{c}$. We denote the $\bar{x}$ as a solution of equation of state $\bar{b}=b_{q}(\bar{x})$. In drawing analogy with the usual van der Waals-Maxwell liquid-gas system, we have here $b_{q}(x)$ the analog of pressure, $\mathrm{x}$ the analog of volume and q the analog of temperature in the liquid-gas system. The detail analysis of phase structure and the related phase transitions for black p-branes is given in [10] and will also be briefly discussed later after (54). In drawing further analogy from the right phase graph in Fig.1, we have the small stable black p-brane phase the analog of the liquid phase while the large stable one the analog of the gas phase in the usual liquid-gas case.
} 

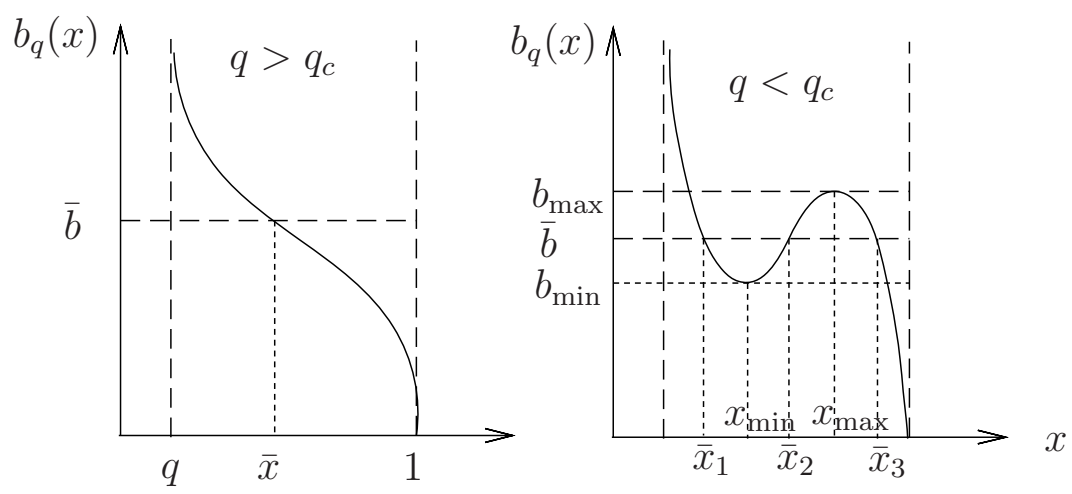

Figure 1: The typical behavior of $b_{q}(x)$ vs $x$ for $q>q_{c}$ and $q<q_{c}$

and $q<q_{c}$ (like the $\tilde{d}>2$ cases), but we don't actually have a critical point in the usual sense and for each subcase the phase structure looks more like that of the $\tilde{d}=1$ case (see [10] for detail). Hence this case can be viewed as a borderline in phase structure which distinguishes the $\tilde{d}=1$ case from the $\tilde{d}>2$ cases. Since $\tilde{d}$ is related to the spatial dimensionality $p$ of the underlying p-branes $(\tilde{d}=D-p-3)$, as an example we take $D=10$ to see their relation. Then the $\tilde{d}>2$ corresponds to charged black p-branes with $p<5$ (Dp and/or F-string when $\mathrm{p}=1), \tilde{d}=2$ corresponds to charged black 5 -branes (D5 or NS5-branes) and $\tilde{d}=1$ corresponds to charged black D6-branes.

Given the above description of phase structure of black p-branes in string/M theory, we have a puzzle here. When the black p-branes with different $p$ (with $1 \leq \tilde{d} \leq 7$ ) are uncharged, they all have the same characteristic phase structure as that of the usual uncharged (Schwarzschild) black hole, having a Hawking-Page transition. However, when they are charged, only those black p-branes with $\tilde{d}>2$ share the same characteristic phase structure of van der Waals-Maxwell liquid-gas type as that of the usual charged (ReissnerNordström) black hole. Such a change of phase structure can be understood as arising from the repulsive interaction due to the charge added to these branes(in addition to the already existing gravitational attraction). But then the natural question would be: why does this kind of change of phase structure not happen for $\tilde{d} \leq 2$ systems? Understanding this dramatic difference in phase structure between $\tilde{d}>2$ cases and $\tilde{d} \leq 2$ cases can probably teach us lessons not only about the universality of van der Waals-Maxwell liquid-gas type phase structure but also the correlation between such universality and the underlying brane interaction. This may also shed some light on the possible underlying holography for such stabilized black hole/brane systems in general if it exists at all. 
While a satisfactory understanding or answer of the above needs our continuing efforts, we expect that a good starting point for this is to explore the underlying cause why the phase structure of $\tilde{d} \leq 2$ systems resists the change when the corresponding brane charges are added5 and whether there exists means which can be used potentially to change qualitatively the phase structure of $\tilde{d} \leq 2$ to that of $\tilde{d}>2$ in canonical ensemble.

As a first step, we have been trying to address the second issue, namely, finding means to change the phase structure of $\tilde{d} \leq 2$ systems to that of the $\tilde{d}>2$ ones (when the branes are charged). Meanwhile we are also accumulating qualitative information and/or evidence for the first one, namely, the underlying cause for the resistance of such a change when charges are added, which we intend to address quantitatively in detail elsewhere.

We mentioned that the charged black branes with their $\tilde{d}=2$ serve as a borderline between the charged black branes with $\tilde{d}=1$ and those with $\tilde{d}>2$ in terms of phase structure and given that fact, it should be much easier to realize such a change if we focus on the $\tilde{d}=2$ branes, which are the charged black D5 branes (or NS5-branes) in $D=10$. We found in a recent publication [1] that the simplest system to realize such a change of phase is to add the delocalized charged D-strings (or F-strings) to the D5 branes (or NS5-branes)6, i.e. to form a D1/D5 (or F/NS5) system. In addition, we find that the delocalized charged black D-strings alone have the exact same phase structure as the charged black D5-branes, without a van der Waals-Maxwell liquid-gas type structure. This would lead one naively to think that when the two are combined, the resulting phase structure should remain the same as before. It turns out that the resulting one is much richer and contains the liquid-gas type, with now a critical line instead of a critical point as in the $\tilde{d}>2$ cases. We find that the physical reason for this change is the existence of a non-vanishing interaction between the delocalized charged black D-strings and charged black D5-branes, which differs from that between the delocalized black Dstrings or between black D5-branes.

However, in the same study we found that the similar thing does not happen for the charged black D6 system (corresponding to $\tilde{d}=1$ ) even if we add delocalized charged D2 branes to form D2/D6 system (Note that both D1/D5 and D2/D6 belong to the same type of $\mathrm{D}(\mathrm{p}-4) / \mathrm{Dp}$ bound state system). Adding the delocalized D4 branes to this system

\footnotetext{
${ }^{5}$ The obvious thing is that unlike the $\tilde{d}>2$ cases, the repulsive interaction due to the added charge in the present case is insufficient to cause the change of the existing phase structure and something more is needed.

${ }^{6}$ Adding the delocalized D3 branes will not work and the resulting phase remains the same as before. This is true also for the general $\mathrm{D}(\mathrm{p}-2) / \mathrm{Dp}$ system. We thank Shibaji Roy for participating this unpublished effort. In the case without the stabilization, this same conclusion was also drawn earlier in [12.
} 
doesn't work, either (see footnote 6 for this case). In the present paper, we explore this further by adding the only remaining lower dimensional branes, namely the delocalized D0 branes, to the charged black D6 system and fortunately, in this case the required change occurs.

Specifically, we consider adding the charged D0 branes to the D6, delocalized along $x^{1}, x^{2}, x^{3}, x^{4}, x^{5}, x^{6}$ of $\mathrm{D} 6$ worldvolume spatial directions. In other words, we consider the system of D0/D6. In $D=10, \mathrm{D} 0$ and D6 are electric-magnetic dual to each other and unlike the bound state D4/D6 or D2/D6, the extremal configuration of D0/D6 preserves no supersymmetry and is actually unstable [13, 14, 15]. But so long the brane (D0 and D6) charges are large enough7, the corresponding bound state is long-lived [15, 16] and therefore it makes sense to consider its thermodynamical phase structure.

One may, at first look, attribute the qualitative change of phase structure of D6 to the added D0 since one would naively think that for D0 branes, the corresponding $\tilde{d}=D-2-d=10-2-1=7>2$. This is actually not the case. For the delocalized charged black D0 branes, their own phase structure (without the presence of D6) is actually the same as that of charged black D6. The same phenomenon was also noticed for the delocalized charged black D-strings and the charged black D5 branes considered in [1]. This would naively suggest that when both D6 and the delocalized D0 are present, the resulting phase structure remain the same as before but this is not the case. Actually, the phase structure turns out to be much richer, containing the van der Waals-Maxwell liquid-gas type, dramatically different from the one when only one type of branes are present. In comparison with the D1/D5 case, the present phase is even richer and more complicated. This is due to the complicated parameter relations of the underlying configuration [14, 15, 17]. Our main purpose here is to reveal the dramatic phase change of charged black D6 when the delocalized charged D0 branes are present and to gain some qualitative information about the relationship between the underlying interaction and the corresponding phase structure. For this, we in this paper consider only a limited case when the D0 brane charge and D6 brane charge are set to equal and the dilaton charge vanishes, for which the parameter relations can be easily solved. We expect to report the full analysis of phase structure of this system and other related things such as the quantitative correlation between the underlying interaction and the phase structure elsewhere.

This paper is organized as follows. In section 2, we present the general configuration of charged black D0/D6 given in [14] in Euclidean signature, discuss the thermodynamical stability and obtain the inverse of local temperature for the purpose of understanding

\footnotetext{
${ }^{7}$ This should be true to validate the gravity configuration.
} 
the underlying phase structure. The details of the underlying phase structure analysis are discussed in section 3. We explain what causes the dramatic change of the phase structure of black D6 branes when delocalized charged D0 are added and conclude this paper in section 4 .

\section{The basic setup}

Our focus in this paper is to see how the added delocalized charged D0 branes (to charged black D6-branes), in the sense described above, change the phase structure of the D6branes qualitatively. For simplicity, we will not consider here the corresponding bubble phase as found relevant in [18] in a similar fashion, which can become globally stable when certain conditions are met.

Let us consider the configuration 8 of charged black D0/D6 [14, 15], now expressed in Euclidean signature as

$$
\begin{aligned}
d s^{2} & =F A^{-\frac{1}{8}} B^{-\frac{7}{8}} d t^{2}+(B / A)^{\frac{1}{8}} \sum_{i=1}^{6} d x_{i}^{2}+A^{\frac{7}{8}} B^{\frac{1}{8}}\left(F^{-1} d \rho^{2}+\rho^{2} d \Omega_{2}^{2}\right) \\
A_{[1]} & =i e^{-3 \phi_{0} / 4} Q\left[\frac{1-\frac{\Sigma}{\rho_{+} \sqrt{3}}}{\rho_{+} B\left(\rho_{+}\right)}-\frac{1-\frac{\Sigma}{\rho \sqrt{3}}}{\rho B(\rho)}\right] d t \\
A_{[7]} & =i e^{3 \phi_{0} / 4} P\left[\frac{1+\frac{\Sigma}{\rho_{+} \sqrt{3}}}{\rho_{+} A\left(\rho_{+}\right)}-\frac{1+\frac{\Sigma}{\rho \sqrt{3}}}{\rho A(\rho)}\right] d t \wedge d x^{1} \ldots \wedge d x^{6} \\
e^{2\left(\phi-\phi_{0}\right)} & =(B(\rho) / A(\rho))^{3 / 2},
\end{aligned}
$$

where the metric is in Einstein frame, each form field is obtained following [8, 10] in such a way that the form field vanishes at the horizon so that it is well defined in the local

\footnotetext{
${ }^{8}$ We actually use the configuration given in [14] with some modifications. The magnetic part of one form $A_{1}$ has now been changed to an electric seven-form potential $A_{7}$ which represents the presence of D6 branes. In $D=10$, D0 branes and D6 branes are electric-magnetic dual to each other. We also express the metric in Einstein-frame for our purpose here. We send the charge parameter $Q, P$ there to $-Q,-P$ and then without loss of generality assume $Q>0, P>0$ for convenience. We correct a typo in the electric one-form potential given in [14] by replacing the dilaton charge $\Sigma$ with $\Sigma / \sqrt{3}$.
} 
inertial frame, $\phi_{0}$ is the asymptotic value of the dilaton, and

$$
\begin{aligned}
& F(\rho)=\left(1-\frac{\rho_{+}}{\rho}\right)\left(1-\frac{\rho_{-}}{\rho}\right), \\
& A(\rho)=\left(1-\frac{\rho_{A+}}{\rho}\right)\left(1-\frac{\rho_{A-}}{\rho}\right), \\
& B(\rho)=\left(1-\frac{\rho_{B+}}{\rho}\right)\left(1-\frac{\rho_{B-}}{\rho}\right),
\end{aligned}
$$

with

$$
\begin{aligned}
\rho_{ \pm} & =M \pm \sqrt{M^{2}+\Sigma^{2}-P^{2} / 4-Q^{2} / 4}, \\
\rho_{A \pm} & =\frac{\Sigma}{\sqrt{3}} \pm \sqrt{\frac{P^{2} \Sigma / 2}{\Sigma-\sqrt{3} M}}, \\
\rho_{B \pm} & =-\frac{\Sigma}{\sqrt{3}} \pm \sqrt{\frac{Q^{2} \Sigma / 2}{\Sigma+\sqrt{3} M}} .
\end{aligned}
$$

In the above, the solutions are characterized by the mass parameter $M$, the delocalized D0 charge parameter $Q$ and the D6 charge parameter $P$. The dilaton charge parameter $\Sigma$ is not independent and is related to $M, Q, P$ via the following

$$
\frac{8}{3} \Sigma=\frac{Q^{2}}{\Sigma+\sqrt{3} M}+\frac{P^{2}}{\Sigma-\sqrt{3} M} .
$$

As noticed in [14], under the electric-magnetic duality, the parameters for the solutions are transformed in the following way

$$
Q \leftrightarrow P, \quad \Sigma \leftrightarrow-\Sigma, \quad M \leftrightarrow M
$$

While the above general configuration is formally given in terms of three parameters $M, P, Q$, it doesn't mean that each set of given values of them, even satisfying (44), will give a well-defined configuration suitable for thermodynamical purpose. For example, it could give a configuration with a naked singularity for certain choice of parameter 9 . For the purpose of the present paper mentioned in the Introduction, we will limit to consider three special cases, namely, 1) the charged black D6-branes, 2) the delocalized charged black D0-branes and 3) the special case of $P=Q$ with vanishing dilaton charge $(\Sigma=0)$,

\footnotetext{
${ }^{9}$ For certain values of $M, Q, P$, either $\rho_{A+}$ or $\rho_{B+}$ can be greater than $\rho_{+}$, then the corresponding configuration will have a naked singularity. For example, for the case of $Q=P$, in addition to the $\Sigma=0$ solution considered in this paper, we have the other two solutions for $\Sigma$ from (4), either of which gives a configuration with a naked singularity, therefore not suitable for thermodynamics.
} 
each of the configurations is well-defined and has a clear meaning of horizon at $\rho=\rho_{+}$. We hope to return to give a full analysis of the underlying phase structure elsewhere for the remaining well-defined configurations with allowed parameters $M, P, Q$ and the suitable solution for dilaton charge $\Sigma$ from (44). For the three special cases just mentioned, each of them in Lorentzian signature has a well-defined horizon at radial coordinate $\rho=\rho_{+}$. Let us consider them each in order:

\subsection{Case 1: $Q=0$}

Let us first bring the configuration given by (1)-(41) in this case to the familiar charged black D6 one [19, 20]. When $Q=0$, we have from (2) as

$$
\begin{aligned}
& F(\rho)=\left(1-\frac{\rho_{+}}{\rho}\right)\left(1-\frac{\rho_{-}}{\rho}\right), \\
& A(\rho)=\left(1+\frac{\Sigma}{\sqrt{3} \rho}\right)\left(1-\frac{\sqrt{3} \Sigma}{\rho}\right), \\
& B(\rho)=\left(1+\frac{\Sigma}{\sqrt{3} \rho}\right)^{2},
\end{aligned}
$$

where we have used (44) and (3). Before we give the explicit $\rho_{ \pm}$, let us examine the expression for the dilaton given in (1) for the present case and we know in a proper radial coordinate $r$ [19, 20]

$$
e^{4\left(\phi-\phi_{0}\right) / 3}=\triangle_{-}(r) \equiv 1-\frac{r_{-}}{r} .
$$

We then have the identifications

$$
r=\rho-\sqrt{3} \Sigma, \quad r_{-}=-\frac{4 \Sigma}{\sqrt{3}}>0,
$$

with a negative dilaton charge as a solution from (4). It is more convenient using parameters $\Sigma, P$ instead of $M, P$. Then we have from (4)

$$
M=\frac{\Sigma}{\sqrt{3}}-\frac{\sqrt{3}}{8} \frac{P^{2}}{\Sigma},
$$

which is supposed to be positive. With this, we have consistently $\rho_{ \pm}$from (3) as

$$
\rho_{+}=\sqrt{3} \Sigma-\frac{\sqrt{3}}{4} \frac{P^{2}}{\Sigma}, \quad \rho_{-}=-\frac{\Sigma}{\sqrt{3}} .
$$

\footnotetext{
${ }^{10}$ Consistency requires $0>\Sigma>-\frac{\sqrt{3}}{4} P$ with $P>0$.
} 
We now use the first equation for the coordinate relation given in (8) to have $r_{ \pm}=$ $\rho_{ \pm}-\sqrt{3} \Sigma$ as

$$
r_{+}=-\frac{\sqrt{3}}{4} \frac{P^{2}}{\Sigma}>0, \quad r_{-}=-\frac{4 \Sigma}{\sqrt{3}}>0,
$$

where the expression for $r_{-}$is just the one given early in (8), therefore a consistent check. Note also that

$$
r_{+} r_{-}=P^{2},
$$

an expected result. In terms of the radial $\mathrm{r}$ coordinate, we have from (13)

$$
\begin{aligned}
& F(r)=\triangle_{+} \triangle_{-}\left(1+\frac{\sqrt{3} \Sigma}{r}\right)^{-2}, \\
& A(r)=\triangle_{-}\left(1+\frac{\sqrt{3} \Sigma}{r}\right)^{-2}, \\
& B(r)=\triangle_{-}^{2}\left(1+\frac{\sqrt{3} \Sigma}{r}\right)^{-2},
\end{aligned}
$$

where

$$
\triangle_{ \pm}=1-\frac{r_{ \pm}}{r},
$$

with $r_{ \pm}$given in (11). We then have the configuration (11) in the present case as

$$
\begin{aligned}
d s^{2} & =\triangle_{-}^{1 / 8}\left(\frac{\triangle_{+}}{\triangle_{-}} d t^{2}+\sum_{i=1}^{6} d x_{i}^{2}\right)+\triangle_{-}^{-7 / 8}\left(\frac{\triangle_{-}}{\triangle_{+}} d r^{2}+r^{2} \triangle_{-}^{2} d \Omega_{2}^{2}\right) \\
A_{[1]} & =0 \\
A_{[7]} & =i e^{3 \phi_{0} / 4} P\left[\frac{1}{r_{+}}-\frac{1}{r}\right] d t \wedge d x^{1} \ldots \wedge d x^{6} \\
e^{2\left(\phi-\phi_{0}\right)} & =\triangle_{-}^{3 / 2},
\end{aligned}
$$

which is just the standard black D6 brane configuration now expressed in Euclidean signature, for example, see [21], with $P=\left(r_{+} r_{-}\right)^{1 / 2}$.

\subsection{Case 2: $\mathrm{P}=0$}

We also want to bring the delocalized charged black D0 brane configuration to the form similar to that given in the previous subsection for the charged black D6 brane one. Given the electric-magnetic relation (5), we expect that for this case the dilaton charge should 
be positive which is true indeed. For this case, we have from (21) as

$$
\begin{aligned}
& F(\rho)=\left(1-\frac{\rho_{+}}{\rho}\right)\left(1-\frac{\rho_{-}}{\rho}\right), \\
& A(\rho)=\left(1-\frac{\Sigma}{\sqrt{3} \rho}\right)^{2}, \\
& B(\rho)=\left(1-\frac{\Sigma}{\sqrt{3} \rho}\right)\left(1+\frac{\sqrt{3} \Sigma}{\rho}\right),
\end{aligned}
$$

where we also use (4) and (3) for the present case. Once again before we give explicit expressions for $\rho_{ \pm}$, we would like to express the configuration in a proper radial coordinate $r$ such that

$$
e^{4\left(\phi-\phi_{0}\right) / 3}=\triangle_{-}^{-1} \equiv\left(1-\frac{r_{-}}{r}\right)^{-1} .
$$

Using the dilaton expression in (1) and the functions $A(\rho)$ and $B(\rho)$ given in (16)), we can read

$$
r=\rho+\sqrt{3} \Sigma, \quad r_{-}=\frac{4 \Sigma}{\sqrt{3}}>0,
$$

with now $\Sigma>0$. One can also see that these two relations can be obtained from (8) by sending $\Sigma \rightarrow-\Sigma$. Once again it is more convenient using parameter $\Sigma, Q$ than $M, Q$. We can solve $M$ in terms of $\Sigma, Q$ from (44) as

$$
M=-\frac{\Sigma}{\sqrt{3}}+\frac{\sqrt{3}}{8} \frac{Q^{2}}{\Sigma}>0 .
$$

With this, we can have consistently 11 from (3)

$$
\rho_{+}=\frac{\sqrt{3}}{4} \frac{Q^{2}}{\Sigma}-\sqrt{3} \Sigma>0, \quad \rho_{-}=\frac{\Sigma}{\sqrt{3}}>0 .
$$

From the first expression given in (18), we have then

$$
r_{+}=\frac{\sqrt{3}}{4} \frac{Q^{2}}{\Sigma}>0, \quad r_{-}=\frac{4 \Sigma}{\sqrt{3}}>0,
$$

where $r_{-}$is just the one given earlier in (18), a consistent check. Note also now that

$$
r_{+} r_{-}=Q^{2}
$$

\footnotetext{
${ }^{11}$ Consistency requires now $0<\Sigma<\sqrt{3} Q / 4$ with $Q>0$.
} 
an expected result. In terms of the radial coordinate $r$, we have from (16)

$$
\begin{aligned}
& F(r)=\triangle_{+} \triangle_{-}\left(1-\frac{\sqrt{3} \Sigma}{r}\right)^{-2}, \\
& A(r)=\triangle_{-}^{2}\left(1-\frac{\sqrt{3} \Sigma}{r}\right)^{-2} \\
& B(r)=\triangle_{-}\left(1-\frac{\sqrt{3} \Sigma}{r}\right)^{-2},
\end{aligned}
$$

where $\triangle_{ \pm}$are given by (14) but now with $r_{ \pm}$given in (21). Then the delocalized charged black D0 brane configuration from (10) is

$$
\begin{aligned}
d s^{2} & =\triangle_{-}^{-1 / 8}\left(\triangle_{+} d t^{2}+\sum_{i=1}^{6} d x_{i}^{2}\right)+\triangle_{-}^{7 / 8}\left(\frac{d r^{2}}{\triangle_{+}}+r^{2} \triangle_{-} d \Omega_{2}^{2}\right) \\
A_{[1]} & =i e^{-3 \phi_{0} / 4} Q\left[\frac{1}{r_{+}}-\frac{1}{r}\right] d t \\
A_{[7]} & =0 \\
e^{2\left(\phi-\phi_{0}\right)} & =\triangle_{-}^{-3 / 2} .
\end{aligned}
$$

This explicit expression, to our knowledge, has not been written down before, which is related to (15) by T-dualities along the isometric directions $x^{i}(i=1,2, \cdots 6)$ with the parameter $P$ replaced by the present $Q$ (Note that the delocalized D0 configuration (24) has the same isometries as the charged black D6 configuration (15)).

\subsection{Case 3: $\mathrm{P}=\mathrm{Q}, \Sigma=0$}

This is the case which we will use to demonstrate how the added delocalized charged D0 branes change the phase structure of charged black D6 branes with $\tilde{d}=1$ dramatically to the one similar to that of charged black p-branes with $\tilde{d}>2$ in canonical ensemble discussed in [10], containing a van-der Waals liquid-gas phase structure. For $P=Q=K$, one of solutions from (44) is $\Sigma=0$, whose extremal one is given in [22]. We have now from (2)

$$
F(r)=\triangle_{-} \triangle_{+}, \quad A(r)=1, \quad B(r)=1,
$$

where we have set $r=\rho$ for the present case and $\triangle_{ \pm}$are once again given in (14) but with now $r_{ \pm}$given as

$$
r_{ \pm}=M \pm \sqrt{M^{2}-K^{2} / 2}
$$


which gives

$$
r_{+} r_{-}=K^{2} / 2
$$

We have now the configuration from (11) as

$$
\begin{aligned}
d s^{2} & =\triangle_{-} \triangle_{+} d t^{2}+\sum_{i=1}^{6} d x_{i}^{2}+\frac{d r^{2}}{\triangle_{-} \triangle_{+}}+r^{2} d \Omega_{2}^{2}, \\
A_{[1]} & =i e^{-3 \phi_{0} / 4} K\left[\frac{1}{r_{+}}-\frac{1}{r}\right] d t \\
A_{[7]} & =i e^{3 \phi_{0} / 4} K\left[\frac{1}{r_{+}}-\frac{1}{r}\right] d t \wedge d x^{1} \ldots \wedge d x^{6} \\
e^{2\left(\phi-\phi_{0}\right)} & =1 .
\end{aligned}
$$

For this configuration, the dilaton remains as a constant and it looks like the usual 4dimensional Reissner-Nordström solution, having two horizons, the outer one at $r=r_{+}$ and inner one at $r=r_{-}$, with a singularity at $r=0$. For the purpose of this paper on phase structure, we are interested only in the outer horizon.

We now return to the general configuration (11) and assume from now on that this configuration is well-defined with a horizon (in Lorentizian signature) at $\rho=\rho_{+}$. For this metric to be free from the conical singularity at the horizon, the Euclidean time coordinate ' $t$ ' must be periodic with periodicity

$$
\beta^{*}=\frac{4 \pi \rho_{+}^{2} \sqrt{A\left(\rho_{+}\right) B\left(\rho_{+}\right)}}{\rho_{+}-\rho_{-}},
$$

the inverse of temperature of the black D0/D6 system at $\rho=\infty$. Here $A\left(\rho_{+}\right), B\left(\rho_{+}\right)$are the ones given in (2) with $\rho=\rho_{+}$. With this, we can read, from the metric, the inverse of local temperature at a given $\rho$ as

$$
\beta=F^{1 / 2} A^{-1 / 16} B^{-7 / 16} \beta^{*}
$$

Applying to the three specific cases considered, we have the inverse of local temperature at a given $r$ for Case 1 ,

$$
\beta=4 \pi r_{+} \triangle_{+}^{1 / 2}(r) \triangle_{-}^{-7 / 16}(r)\left(1-\frac{r_{-}}{r_{+}}\right)^{1 / 2}=4 \pi \bar{r}_{+} \triangle_{-}^{-1}(\bar{r}) \triangle_{+}^{1 / 2}(\bar{r})\left(1-\frac{\bar{r}_{-}}{\bar{r}_{+}}\right)^{1 / 2}
$$

for Case 2,

$$
\beta=4 \pi r_{+} \triangle_{-}^{-1 / 16}(r) \triangle_{+}^{1 / 2}(r)\left(1-\frac{r_{-}}{r_{+}}\right)^{1 / 2}=4 \pi \bar{r}_{+} \triangle_{-}^{-1}(\bar{r}) \triangle_{+}^{1 / 2}(\bar{r})\left(1-\frac{\bar{r}_{-}}{\bar{r}_{+}}\right)^{1 / 2}
$$


and for case 3 ,

$$
\beta=4 \pi r_{+} \triangle_{-}^{1 / 2}(r) \triangle_{+}^{1 / 2}(r)\left(1-\frac{r_{-}}{r_{+}}\right)^{-1}=4 \pi \bar{r}_{+} \triangle_{-}^{1 / 2}(\bar{r}) \triangle_{+}^{1 / 2}(\bar{r})\left(1-\frac{\bar{r}_{-}}{\bar{r}_{+}}\right)^{-1}
$$

where for each case in the last equality, we have used the so-called physical radius $\bar{r}$ (rather than the coordinate one $r$ ) via $\bar{r}=r \triangle_{-}^{9 / 16}$ for Case $1, \bar{r}=r \triangle_{-}^{15 / 16}$ for Case 2 , and $\bar{r}=r$ for Case 3, respectively, from their corresponding metric. In the above, we also define the physical parameters $\bar{r}_{ \pm}$in the same way for each case and the corresponding $\triangle_{ \pm}(\bar{r})$ remains in form the same as before,

$$
\triangle_{ \pm}=1-\frac{r_{ \pm}}{r}=1-\frac{\bar{r}_{ \pm}}{\bar{r}}
$$

To study the equilibrium thermodynamics [23] in canonical ensemble, the allowed configuration (say, the black D0/D6 system or its extremal one) should be placed in a cavity with a fixed radius $\bar{r}_{B}\left(>\bar{r}_{+}\right)$for the reason as explained in the Introduction [7]. The other fixed quantities are the cavity temperature $1 / \beta$, the physical periodicity of each $x^{i}$ with $12 i=1, \cdots 6$, the dilaton value $\bar{\phi}$ on the surface of the cavity $\left(\right.$ at $\bar{r}=\bar{r}_{B}$ ) and the charges/fluxes enclosed in the cavity $\bar{Q}_{p}(p=0,6)$, respectively. In equilibrium, these fixed values are set equal to the corresponding ones of the allowed configuration enclosed in the cavity. For example, we set the charge

$$
\bar{Q}_{p}=Q_{p} \equiv \frac{i}{\sqrt{2} \kappa} \int e^{-a(d) \phi} * F_{[d+1]},
$$

for $d=p+1=1,7$, respectively. In the above, $*$ denotes the Hodge duality and the field strength $F_{[d+1]}=d A_{[d]}$ with $A_{[d]}$ the corresponding form potential. With the potentials $A_{1}, A_{7}$ given in (11), we have

$$
\begin{aligned}
& F_{2}=i e^{-3 \phi_{0} / 4} Q \frac{A(\rho)}{\rho^{2} B^{2}(\rho)} d \rho \wedge d t \\
& F_{8}=i e^{3 \phi_{0} / 4} P \frac{B(\rho)}{\rho^{2} A^{2}(\rho)} d \rho \wedge d t \wedge d x^{1} \wedge \cdots \wedge d x^{6},
\end{aligned}
$$

where the explicit expressions for $A(\rho)$ and $B(\rho)$ are given in (2), respectively. We therefore have the D0-brane charge and D6-brane charge per unit six-brane volume, respectively, as

$$
Q_{0}=\frac{\Omega_{2} Q}{\sqrt{2} \kappa} e^{3 \phi_{0} / 4} V_{6}^{*}, \quad Q_{6}=\frac{\Omega_{2} P}{\sqrt{2} \kappa} e^{-3 \phi_{0} / 4}
$$

\footnotetext{
${ }^{12}$ Note that to have a finite Euclidean action for the D0/D6 system, the brane coordinates $x^{i}$ with $i=1, \cdots 6$ should be compact.
} 
where $\Omega_{n}$ denotes the volume of a unit $n$-sphere, $V_{6}^{*} \equiv \int d x^{1} d x^{2} \cdots d x^{6}$ is the coordinate volume, and $\kappa$ is a constant with $1 /\left(2 \kappa^{2}\right)$ appearing in front of the Hilbert-Einstein action in canonical frame but containing no asymptotic string coupling $g_{s}=e^{\phi_{0}}$. For the consideration of phase structure, as usual, we need to express these charges in each case in terms of their fixed or/and physical quantities on the cavity. We have for Case 1,

$$
Q_{0}=0, \quad Q_{6}=\frac{\Omega_{2}}{\sqrt{2} \kappa} e^{-3 \bar{\phi} / 4}\left(\bar{r}_{+} \bar{r}_{-}\right)^{1 / 2}
$$

where we have used the dilaton formula in (15) to express its asymptotic value $\phi_{0}$ in terms of its fixed value $\bar{\phi}$ at $\bar{r}=\bar{r}_{B}$ and use the physical parameter $\bar{r}_{ \pm}$in replace of $P$ via (12), for Case 2,

$$
Q_{0}=\frac{\Omega_{2} \bar{V}_{6}}{\sqrt{2} \kappa} e^{3 \bar{\phi} / 4}\left(\bar{r}_{-} \bar{r}_{+}\right)^{1 / 2}, \quad Q_{6}=0,
$$

where we have used $\bar{\phi}$ in replace of $\phi_{0}$ via (24) and the physical parameter $\bar{r}_{ \pm}$in replace of $Q$ via (22), respectively, and the physical volume $\bar{V}_{6}=\triangle_{-}^{-3 / 8} V_{6}^{*}$, and for Case 3 ,

$$
Q_{0}=\frac{\Omega_{2} \bar{V}_{6}}{\sqrt{2} \kappa} e^{3 \bar{\phi} / 4}\left(2 \bar{r}_{-} \bar{r}_{+}\right)^{1 / 2}, \quad Q_{6}=\frac{\Omega_{2}}{\sqrt{2} \kappa} e^{-3 \bar{\phi} / 4}\left(2 \bar{r}_{-} \bar{r}_{+}\right)^{1 / 2},
$$

where now $\bar{\phi}=\phi_{0}, \bar{V}_{6}=V_{6}^{*}$ and we have expressed $P=Q=K$ in terms of $\bar{r}_{ \pm}$using (27) (note here $\bar{r}_{ \pm}=r_{ \pm}$).

In the above, we have expressed all the quantities in terms of their fixed (or physical) correspondences, for example, we have replaced the asymptotical string coupling $g_{s}$ in terms of the fixed effective string coupling on the cavity via $e^{\bar{\phi}}=e^{\phi\left(\bar{r}_{B}\right)}$ in each case. In canonical ensemble, it is the Helmholtz free energy which determines the stability of equilibrium states and is related to the Euclidean action by $F=I_{E} / \beta$ in the leading order approximation. The procedure for evaluating the Euclidean action of black $p$-branes was given in detail in [10] following the standard technique. The generalization to the present case is straightforward, similar to what we did for the $\mathrm{D}(\mathrm{p}-4) / \mathrm{Dp}$ systems in [1], though the computation is a bit lengthy, by considering one more piece contribution from the form field strength $F_{2}$ and its potential $A_{1}$ in addition to that from the usual $F_{8}$ and its potential $A_{7}$ for black D6. The explicit expression of action for each relevant case will be given in the following section and it can be used to analyze the underlying phase structure. What is important is the recognition that for given cavity temperature $1 / \beta$, the Euclidean action is essentially the free energy $\left(I_{E}=\beta F\right)$. So the minimum of free energy is that of the Euclidean action. Hence the minimum of Euclidean action will determine the local stability of the underlying system under consideration. Note that in canonical 
ensemble, the only variable is the horizon size $\bar{r}_{+}$and we always have 13

$$
\frac{d I_{E}\left(\bar{r}_{+}\right)}{d \bar{r}_{+}} \sim\left(\beta-\beta\left(\bar{r}_{+}\right)\right)
$$

and

$$
\frac{d I_{E}\left(\bar{r}_{+}\right)}{d \bar{r}_{+}}=0 \Rightarrow \beta\left(\bar{r}_{+}\right)=\beta,
$$

where the extremal condition of $I_{E}$ is nothing but the thermal equilibrium of the system inside cavity with the cavity with a preset temperature $1 / \beta$. In the above, we have computed the respective $\beta\left(\bar{r}_{+}\right)$using the corresponding explicit Euclidean action given in the following section and find that it is nothing but the inverse of local temperature at $\bar{r}=\bar{r}_{B}$ for each case given earlier in (31), (32) and (33), respectively, therefore a consistent check. At the solution of $\beta\left(\bar{r}_{+}\right)=\beta$ given above, we have

$$
\frac{d^{2} I_{E}\left(\bar{r}_{+}\right)}{d \bar{r}_{+}^{2}} \sim-\frac{d \beta\left(\bar{r}_{+}\right)}{d \bar{r}_{+}},
$$

so the minimum of $I_{E}$ at the solution, i.e., $d^{2} I_{E} / d \bar{r}_{+}^{2}>0$, requires the negative slope of $\beta\left(\bar{r}_{+}\right)$there. Therefore, as always, the function $\beta\left(\bar{r}_{+}\right)$computed above or given earlier is the key for us to determine the phase structure of underlying system which we will discuss in the following section. The above stability discussion can be verified for each case considered with the corresponding explicitly-evaluated Euclidean action given in the following section and the key function $\beta\left(\bar{r}_{+}\right)$for phase structure can also be obtained accordingly.

\section{The analysis of phase structure}

In this section, we will give the phase structure of each case considered in the previous sections, in particular, Case 3 which is the focus of this paper. Let us now discuss each of them in order.

\subsection{Case 1: $\mathrm{Q}=0$}

This is just the usual charged black D6 brane and the inverse of local temperature given in (31) is nothing but the one given in [10]. The phase structure of this system, i.e, the

\footnotetext{
${ }^{13}$ Another way to understand this is $I_{E}\left(r_{+}\right)=\beta F=\beta E\left(r_{+}\right)-S\left(r_{+}\right)$where $E\left(r_{+}\right)$is the internal energy and $S\left(r_{+}\right)$the entropy. In canonical ensemble, we have fixed cavity size, temperature and the charge inside, so $d E=T\left(r_{+}\right) d S$. We then have $d I_{E} / d r_{+}=\beta d E / d r_{+}-d S / d r_{+}=T\left(r_{+}\right)\left(d S / d r_{+}\right)\left(\beta-\beta\left(r_{+}\right)\right)$ with $\beta\left(r_{+}\right)=1 / T\left(r_{+}\right)$. Note that $S$ is the only function of $r_{+}$and $d S / d r_{+}>0$, therefore we have (42).
} 
$\tilde{d}=1$ case, was given there, similar to the one for chargeless black p-branes, containing no the van-der Waals-Maxwell liquid-gas type. We will refer this case to [10] for detail.

\subsection{Case 2: $\mathrm{P}=0$}

This is the first new case encountered in this paper. The delocalized charged black D0 branes alone would at first look give a rather different phase structure than the charged black D6 branes since the localized charged black D0 branes seemly correspond to the $\tilde{d}=7(\tilde{d}=D-d-2=10-1-2=7)$ case discussed in [10], containing the van der Waals-Maxwell liquid-gas phase structure. However, just like the delocalized D-strings in the case of D1/D5 discussed very recently in [1], the present delocalized black D0 branes once again turn out to share the same phase structure as the charged black D6 branes, containing actually no van der Waals-Maxwell liquid-gas type. This can be seen from the inverse of local temperature in canonical ensemble for the present case given in (32) which is identical to the one for the charged black D6 branes as given in (31). This can also be seen from its explicit reduced Euclidean action

$$
\begin{aligned}
\tilde{I}_{E} & \equiv \frac{2 \kappa^{2} I_{E}}{4 \pi \Omega_{2} \bar{V}_{6} \bar{r}_{B}^{2}} \\
& =\frac{\beta}{4 \pi \bar{r}_{B}}\left[-3 \sqrt{\frac{\triangle_{+}}{\triangle_{-}}}-\sqrt{\triangle_{+} \triangle_{-}}+4\right]-\left(\frac{\bar{r}_{+}}{\bar{r}_{B}}\right)^{2} \triangle_{-}^{-3 / 2}\left(1-\frac{\bar{r}_{-}}{\bar{r}_{+}}\right)^{3 / 2},
\end{aligned}
$$

which is identical in form to the correspondence of charged black D6 branes given in [10] and from which the same $\beta\left(\bar{r}_{+}\right)$as given in (32) can be obtained following the prescription given in the previous section. This is a bit surprise since the metric as given in (24) for the delocalized charged black D0 branes looks completely different from that given in (15) for charged black D6 branes, not mentioning two types of different objects. One of possible reasons for the two sharing the same phase structure is that from our experience on the black p-branes given in [10], the underlying phase structure depends crucially on the dimensionality of transverse dimensions and for both of these cases this dimensionality is indeed the same. The other more sounding reason is that these two configurations are related to each other by T-dualitie 14 along the isometric $x^{i}$ directions with $i=1, \cdots 6$,

\footnotetext{
${ }^{14}$ Showing this is straightforward, following [24, 25, 26]. We stress that an isometric direction transverse to a black brane cannot be generated by placing an infinite periodic array of such black branes along this direction since unlike the BPS case we do not have the so-called "no-force" condition, crucial for this purpose, for black branes placed parallel to each other. In general, one has to solve the corresponding equations of motion for such delocalized black brane configuration with certain isometries for performing T-dualities along these directions, see [26] for example. Two black configurations can be T-dual related
} 
therefore are T-dual equivalent(Note that the delocalized black D0 brane configuration (24) has the same isometries as the charged black D6 brane configuration (15D) and Tduality keeps the underlying phase structure unchange.

\subsection{Case 3: $\mathrm{P}=\mathrm{Q}=\mathrm{K}$}

This case is the main focus of this paper reflecting the dramatic change of phase structure of the charged black D6 branes when the delocalized charged D0 branes are added. The discussion of the previous two subsections seemly suggests that when the delocalized charged D0 branes and charged D6 branes are combined to form the charged black D0/D6 system, the resulting phase structure would be supposed to remain the same as that when either type of the branes are present. This actually happens when the delocalized D4 branes are added to the charged black D6 branes as mentioned in footnote 6 and this remains almost true when the delocalized D2 branes are added 15 . The only remaining lower dimensional objects available for our purpose are the delocalized D0 branes. Just like the added delocalized D-strings to the charged black D5 branes, the added delocalized D0-branes finally change the phase structure of charged black D6 branes to the one similar to that of black p-branes with $\tilde{d}>2$, containing a van der Waals-Maxwell liquid-gas type. Let us examine this in what follows.

The key quantity as mentioned at the end of last section is the inverse of local temperature $\beta\left(r_{+}\right)$given in (33). Before discussing the behavior of this function, we address a few related issues. This case is $P=Q=K$ and from (41) and (27) we need to set

$$
\frac{\bar{Q}_{0}}{\bar{V}_{6}}=\bar{Q}_{6} e^{3 \bar{\phi} / 2},
$$

where we have set $Q_{p}=\bar{Q}_{p}$ for $p=0,6$, respectively. Note that $\bar{Q}_{0}, \bar{Q}_{6}, \bar{\phi}$ and $\bar{V}_{6}$ are all fixed in the canonical ensemble and for the present case they need to satisfy the above relation. Using (41), we define a reduced fixed charge with a dimension of length as

$$
\tilde{Q}=\frac{\kappa \bar{Q}_{6} e^{3 \bar{\phi} / 4}}{\Omega_{2}}=\frac{\kappa \bar{Q}_{0} e^{-3 \bar{\phi} / 4}}{\bar{V}_{6} \Omega_{2}} .
$$

With this, we have from (41)

$$
\bar{r}_{-}=\frac{\tilde{Q}^{2}}{\bar{r}_{+}}
$$

to each other only when the two configurations have the same isometries.

${ }^{15}$ When the delocalized charged D2 branes are added to the charged black D6 branes [1], the resulting phase has been changed to one similar to that of the charged black D5 even though the basic phase structure is still the one similar to the chargeless case. 
which indicates that $\bar{r}_{-}$is not independent and is related to $\bar{r}_{+}$via the above. For simplicity of further discussion, as usual, we define the so-called reduced quantities at the fixed radius $\bar{r}=\bar{r}_{B}$,

$$
x \equiv \frac{\bar{r}_{+}}{\bar{r}_{B}}<1, \quad \bar{b} \equiv \frac{\beta}{4 \pi \bar{r}_{B}}, \quad q \equiv \frac{\tilde{Q}}{\bar{r}_{B}}<x .
$$

Note that $0<q<1$ (since $x<1)$ and $q<x<1$ ( since $\bar{r}_{+}>\bar{r}_{-}$). With these, we have

$$
\triangle_{-}=1-\frac{\bar{r}_{-}}{\bar{r}_{B}}=1-\frac{q^{2}}{x}, \quad \triangle_{+}=1-\frac{\bar{r}_{+}}{\bar{r}_{B}}=1-x .
$$

At $\bar{r}=\bar{r}_{B}$, the inverse of local temperature (33) for the present case is

$$
\beta\left(\bar{r}_{+}\right)=4 \pi \bar{r}_{+} \triangle_{-}^{1 / 2}\left(\bar{r}_{B}\right) \triangle_{+}^{1 / 2}\left(\bar{r}_{B}\right)\left(1-\frac{\bar{r}_{-}}{\bar{r}_{+}}\right)^{-1} .
$$

Therefore, from the above, the corresponding inverse of reduced local temperature is

$$
b_{q}(x) \equiv \frac{\beta\left(r_{+}\right)}{4 \pi r_{B}}=\frac{x(1-x)^{1 / 2}\left(1-\frac{q^{2}}{x}\right)^{1 / 2}}{1-\frac{q^{2}}{x^{2}}} .
$$

This same $b_{q}(x)$ can also be obtained from the corresponding reduced Euclidean action given below, following the prescription given in the previous section,

$$
\begin{aligned}
\tilde{I}_{E} & \equiv \frac{2 \kappa^{2} I_{E}}{4 \pi \Omega_{2} \bar{V}_{6} \bar{r}_{B}^{2}} \\
& =-4 \frac{\beta}{4 \pi \bar{r}_{B}}\left(\sqrt{\triangle_{-} \triangle_{+}}-1\right)-\left(\frac{\bar{r}_{+}}{\bar{r}_{B}}\right)^{2}, \\
& =-4 \bar{b}\left(\sqrt{(1-x)\left(1-\frac{q^{2}}{x}\right)}-1\right)-x^{2},
\end{aligned}
$$

where in the last equality we have used (49) and (50). Given the above form of $b_{q}(x)$, we have

$$
b_{q}(x \rightarrow q) \rightarrow \infty, \quad b_{q}(x \rightarrow 1) \rightarrow 0,
$$

which is the characteristic behavior of the corresponding $b_{q}(x)$ for black p-branes with $\tilde{d}>2$. Our experience tells that the underlying phase structure contains the van der Waals-Maxwell liquid-gas type and there exists a critical charge $q_{c}$. When $q>q_{c}$, the equation of state $\bar{b}=b_{q}(\bar{x})$ has a unique solution $\bar{x}$ which gives a stable phase. When $q=q_{c}$, we have a second-order phase transition point, a critical point, at which we have a critical size $x_{c}$ and a critical $b_{c}$ or critical temperature $T_{c}=1 /\left(4 \pi r_{B} b_{c}\right)$ and where there is no entropy change during the phase transition. When $q<q_{c}, b_{q}(x)$ has a maximum and 
a minimum, occurring at $x_{\max }$ and $x_{\min }$, respectively, with $0<x_{\min }<x_{\max }<1$. When the given $\bar{b}$ is between the minimum and the maximum, the equation of state $b_{q}(\bar{x})=\bar{b}$ has three solutions $\bar{x}_{1}<\bar{x}_{2}<\bar{x}_{3}$ but only $\bar{x}_{1}$ and $\bar{x}_{3}$ give the locally stable phases, respectively, while $\bar{x}_{2}$ gives a unstable one since $b_{q}(x)$ has a positive slope at $\bar{x}=\bar{x}_{2}$. For each given $q<q_{c}$, there always exists a $b_{t}(q)$, which is a function of charge $q$ only, for which the corresponding phase at $\bar{x}_{1}$ and the one at $\bar{x}_{3}$ have the same free energy, therefore can coexist. The corresponding phase transition is a first-order one since it involves an entropy change during the phase transition (Note that the entropy is a function of $x$ and the transition involves a change of size $x$ ). Since $q<q_{c}$ is a one-parameter line segment, we therefore have a line of first-order phase transition terminating at the above mentioned second-order critical point. For given $q<q_{c}$, when $\bar{b}>b_{t}(q)$, the small size $\bar{x}_{1}$ phase has a lower free energy, therefore more stable while when $\bar{b}<b_{t}(q)$, on the other hand the large size $\bar{x}_{3}$ phase is more stable. The underlying phase is nothing but that of van der Waals-Maxwell liquid-gas type and in drawing the analogy, the small-size phase is like liquid one while the large size one like the gas phase. This behavior has been discussed in detail in [10] for charged black p-branes with $\tilde{d}>2$ and it applies here, too.

Let us go a bit further about the behavior of $b_{q}(x)(\underline{52})$. The $x_{\min }$ and $x_{\max }$ mentioned above are determined by $d b_{q}(x) / d x=0$, which gives

$$
x^{4}-\frac{2}{3}\left(1+q^{2}\right) x^{3}-2 q^{2} x^{2}+2 q^{2}\left(1+q^{2}\right) x-\frac{5}{3} q^{4}=0 .
$$

This is a quartic equation and has four roots in general. Since the sign of the last term (the constant term) in the equation is negative, therefore the number of negative roots is one or three. Note that $q<x<1$ and if we use $f(x)$ to represent the left-hand side of the above equation, we have $f(q)=4 q^{2}(1-q)^{2} / 3>0$ and $f(1)=\left(1-q^{2}\right)^{2} / 3>0$, both of which are positive. So if $f(x)=0$, i.e. (55), has any solution in the region of $q<x<1$, the number of them must be two. The function $f(x)$ has a unique minimum in the region of $q<x<1$. We can show this by setting its first derivative vanish and this gives the equation

$$
x^{3}-\frac{1+q^{2}}{2} x^{2}-q^{2} x+\frac{1+q^{2}}{2} q^{2}=0,
$$

which has three solutions $x=-q, q$ and $\left(1+q^{2}\right) / 2$, among which only the last solution falls in the region of interest. Further if we denote $f^{\prime \prime}(x)$ as the second derivative of $f(x)$, we have

$$
f^{\prime \prime}\left(\frac{1+q^{2}}{2}\right)=\frac{\left(1-q^{2}\right)^{2}}{4}>0
$$

therefore $f(x)$ has a unique minimum $f_{\text {min }}$ occurring at $x=\left(1+q^{2}\right) / 2$, which implies that $f(x)=0$ or (55) has either two roots when $f_{\min }<0$ or none when $f_{\min }>0$ in the region 
of $q<x<1$. The value of $f_{\min }$ can be evaluated as

$$
f_{\min }=-\frac{1}{48}\left(1-q^{2}\right)^{2}\left[1+2 \sqrt{5} q+q^{2}\right](\sqrt{5}+2-q)(\sqrt{5}-2-q) .
$$

Given $0<q<1, f_{\min }>0$ requires $q>\sqrt{5}-2$ with which (55) has no solutions in the region of $q<x<1$, therefore $b_{q}(x)$ decreases monotonically from the behavior of $b_{q}(x)$ given in (54). So the equation of state $\bar{b}=b_{q}(\bar{x})$ has a unique solution $\bar{x}$ for each given $\bar{b}$ and the slope of $b_{q}(x)$ at $x=\bar{x}$ is negative, therefore giving a stable phase. $f_{\min }<0$ requires $q<\sqrt{5}-2$ and now (55) has two solutions $x_{\min }<x_{\max }$ in the region of $q<x<1$ once again given the behavior of $b_{q}(x)$ (54), the former giving the minimum of $b_{q}(x)$ and the latter the maximum. For each given $\bar{b}$ between the minimum and the maximum of $b_{q}(x)$, the equation of state $\bar{b}=b_{q}(\bar{x})$ has three solutions $\bar{x}_{1}<\bar{x}_{2}<\bar{x}_{3}$ and exactly following the discussion given in [10], we will have a van der Waals-Maxwell phase structure as described earlier and will not repeat it here. $f_{\min }=0$ requires $q=q_{c}=\sqrt{5}-2$ at which the two roots of (55) $x_{\min }$ and $x_{\max }$ coincide and so $x_{\min }=x_{\max }=x_{c}=\left(1+q_{c}^{2}\right) / 2=5-2 \sqrt{5}$. Now the phase transition involves no entropy change and therefore it is a second-order critical point. At this point, not only we have the vanishing first derivative of $b_{q}(x)$, its second derivative vanishes, too. The critical parameters can be collectively given as

$$
q_{c}=\sqrt{5}-2, \quad x_{c}=5-2 \sqrt{5}, \quad b_{c}=\frac{5^{5 / 4}(\sqrt{5}-2)^{3 / 2}}{2},
$$

where $b_{c}$ is obtained by substituting $x_{c}$ and $q_{c}$ into $b_{q}(x)$. The above critical charge can also be obtained following [10] from the discriminant of (55) as

$$
\triangle=\frac{256}{27} q^{6}\left(q^{2}-1\right)^{4}\left[(q-2)^{2}-5\right]\left[(q+2)^{2}-5\right],
$$

which vanishes only at $q_{c}=\sqrt{5}-2$ (since $0<q<1$ ), implying the two positive roots being identical. The characteristic behaviors, described above, of function $f(x)$ and $b_{q}(x)$ for $q>q_{c}, q=q_{c}$ and $q<q_{c}$ are given in Fig. 2 and Fig. 3, respectively. Note that we choose a smaller $q$ in Fig. 3 than the one in Fig. 2 in the case of $q<q_{c}$ so that the $b_{q}(x)$ curve shows clearly a minimum and a maximum.

Due to the complication of the general configuration (11) for charged black D0/D6 system, we use its two special cases, namely, the $P=0$ case and the $P=Q$ case to demonstrate: 1) the delocalized D0 branes alone have the same phase structure as the charged black D6 branes, and 2) adding the delocalized D0 branes to the charged black D6 branes changes the phase structure of the latter dramatically to the one, now containing a van der Waals-Maxwell liquid-gas type structure, which does not occur when either the delocalized D2 or D4 branes are added. It has long been known (also obvious from the 


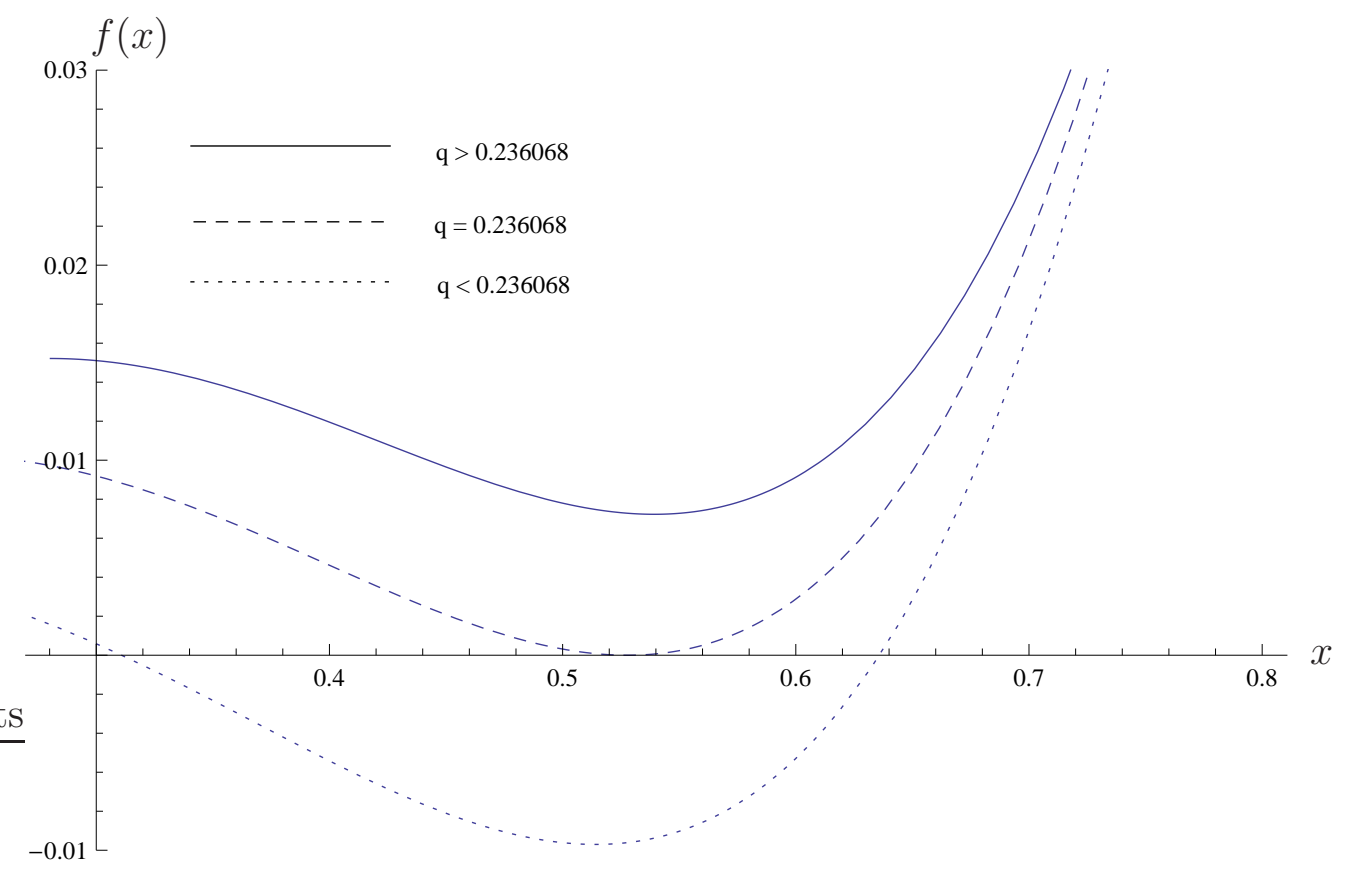

Figure 2: The characteristic behaviors of $f(x)$ vs $x$ for $q>q_{c}, q=q_{c}$ and $q<q_{c}$ with $q_{c}=\sqrt{5}-2 \approx 0.236068$ as described in the text, respectively.

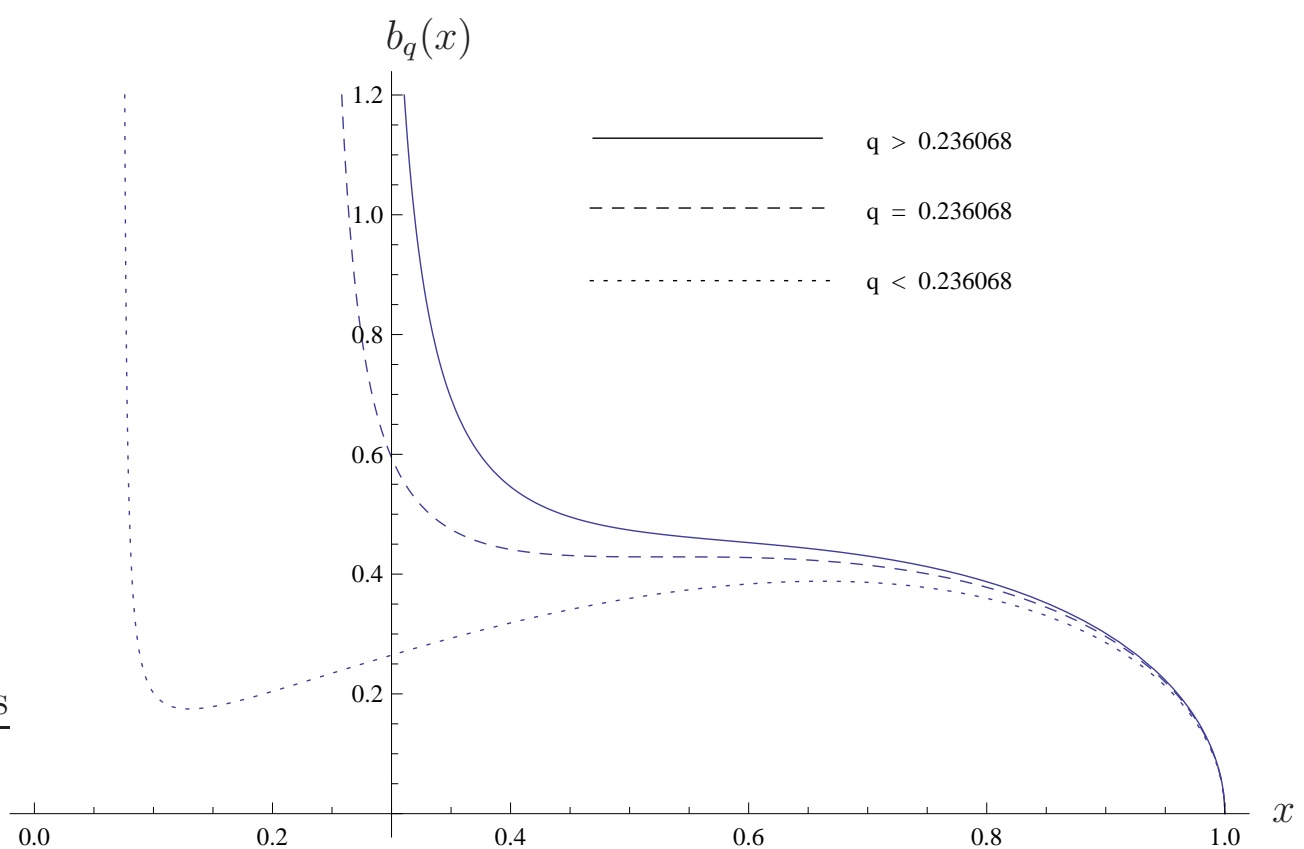

Figure 3: The characteristic behaviors of $b_{q}(x)$ vs $x$ for $q>q_{c}, q=q_{c}$ and $q<q_{c}$ with $q_{c}=\sqrt{5}-2 \approx 0.236068$ as described in the text, respectively. 
metric given in (28)) that the special $P=Q$ configuration, when dimensionally reduced along D6 worldvolume directions from $D=10$ to $D=4$, is nothing but the $D=4$ Reissner-Nordström one and its phase structure in canonical ensemble has been analyzed in [2, 3], though using a rather different way from what we did above. It is interesting to find that the inverse of local temperature $\beta\left(r_{+}\right)$used there is same as ours (33) and so the phase structure is exactly the same though the purpose here (showing how the added delocalized D0 branes change qualitatively the phase structure of charged black D6 branes dramatically) is completely different from theirs. Nevertheless this reflects that the phase structure will remain the same for dimensionally-reduced related systems, an expected result, in addition to what we have found about T-dual related systems.

\section{Discussion and conclusion}

In this paper, we explore means which can be used potentially to change or modulate qualitatively the phase structure of black p-branes in canonical ensemble. Following [10], we know that the charged black branes with their $\tilde{d}=2$ serve as a borderline between the black branes with $\tilde{d}=1$ and those with $\tilde{d}>2$ in phase structure. In a previous paper [1], we have studied the $\tilde{d}=2$ case in $D=10$ and the corresponding branes are charged black D5 branes. The delocalized charged black D-strings alone are found to have the exact same phase structure as the charged black D5-branes without a van-der Waals-Maxwell liquid-gas type structure. But when the two are combined to form D1/D5 system, the resulting phase is much richer and contains the liquid-gas type. We find that the physical reason for this to occur is the existence of a non-vanishing interaction between charged black D-strings and charged black D5-branes, which differs from that between the delocalized black D-strings or between black D5-branes. However, for the same type of D2/D6 system, we don't find that adding the delocalized D2 branes to the charged black D6 branes changes the phase structure of charged black D6 branes that much and actually this only changes the phase structure of latter to the one similar to that of charged black D5 branes, still containing no van der Waals-Maxwell liquid-gas type.

In general, adding delocalized $\mathrm{D}(\mathrm{p}-2)$ branes to Dp branes (see footnote 6) will not change the phase structure of the latter at all. The only remaining possibility for this purpose is to consider adding the delocalized charged D0 branes to the charged black D6 branes and it turns out that in this case, the phase structure get modified to the required one. Note that as discussed in this paper the delocalized charged black D0 branes, D2 branes or D4 branes each alone have the same phase structure as the charged black D6 
branes with $\tilde{d}=1$ described in detail in [10] (similar to the one for the chargeless case). As discussed in the previous section, one possible reason for this is that each of them have the same transverse dimensions $\tilde{d}+2=3$ and the other more sounding reason is that they are all related to each other by T-dualities (They all have the same isometries). However, when each of them are combined with charged D6 branes to form the corresponding charged black bound state $\mathrm{D}(6-\mathrm{n}) / \mathrm{D} 6$ with $n=2,4,6$, the resulting phase structures are rather different, a bit of surprise. When $n=2$, the phase structure of the charged black D4/D6 remains the same as that of individual constituents, the naively expected one. When $n=4$, the resulting phase structure of the charged black D2/D6 is changed to the one similar to that of charged black D5 branes with $\tilde{d}=2$ given in [10, 1], but in essence the phase structure is still in the same class as that for the chargeless case as described earlier, containing no van der Waals-Maxwell liquid-gas type. Only for $n=6$, the resulting phase structure got changed dramatically to the one similar to that of charged black p-branes with $\tilde{d}>2$, containing now a van der Waals-Maxwell liquid-gas type as described in the previous section. We all know (for example, see [27) that when $\mathrm{D}(6$ n) and D6 are combined to form charged black bound state $\mathrm{D}(6-\mathrm{n}) / \mathrm{D} 6$, the interaction between constituent $\mathrm{D}(6-\mathrm{n})$ and $\mathrm{D} 6$ are different for different $n=2,4,6$, respectively. For example, adding the delocalized charged D4 to D6 gives rise to an additional attractive interaction while adding delocalized charged D0 to D6 gives rise to an additional repulsive interaction. As mentioned earlier, the repulsive interaction due to the added D6 brane charge is not sufficient to change its existing phase structure and additional repulsive interaction is needed for this purpose. Therefore it is not surprised for us to have the above phase structure for the corresponding system at least qualitatively. So the above different phase structure for each case should be expected qualitatively to be due to the different interaction between the constituent branes. It would be interesting to explore the exact or quantitative correlation between the interaction and the phase structure and we hope to return this issue elsewhere. We also find that the black systems related by T-duality (to be precise, that two black systems are related by T-duality here means that the corresponding two gravity configurations can be related to each other by T-duality in the sense described earlier and they must have the same isometries. For example, the delocalized charged black D0 configuration (24) is T-dual to the charged black D6 configuration (15).) or dimensionally-reduced black related systems have the same phase structure. Though expected, it is nice to confirm this. 


\section{Acknowledgements:}

We would like to thank Shibaji Roy for help in polishing the revision. We acknowledge support by grants from the Chinese Academy of Sciences and grants from the NSF of China with Grant No : 10975129 and 11235010.

\section{References}

[1] J. X. Lu, R. Wei and J. Xu, "The phase structure of black D1/D5 (F/NS5) system in canonical ensemble," JHEP 1212, 012(2012). arXiv:1210.0708 [hep-th].

[2] S. Carlip and S. Vaidya, "Phase transitions and critical behavior for charged black holes," Class. Quant. Grav. 20 (2003) 3827 [arXiv:gr-qc/0306054].

[3] A. P. Lundgren, "Charged black hole in a canonical ensemble," Phys. Rev. D 77, 044014 (2008) arXiv:gr-qc/0612119.

[4] S. W. Hawking and D. N. Page, "Thermodynamics Of Black Holes In Anti-De Sitter Space," Commun. Math. Phys. 87, 577 (1983).

[5] A. Chamblin, R. Emparan, C. V. Johnson and R. C. Myers, "Charged AdS black holes and catastrophic holography," Phys. Rev. D 60, 064018 (1999) arXiv:hep-th/9902170.

[6] A. Chamblin, R. Emparan, C. V. Johnson and R. C. Myers, "Holography, thermodynamics and fluctuations of charged AdS black holes," Phys. Rev. D 60, 104026 (1999) arXiv:hep-th/9904197.

[7] J. W. York, "Black hole thermodynamics and the Euclidean Einstein action," Phys. Rev. D 33, 2092 (1986).

[8] H. W. Braden, J. D. Brown, B. F. Whiting and J. W. . York, "Charged black hole in a grand canonical ensemble," Phys. Rev. D 42, 3376 (1990).

[9] E. Witten, "Anti-de Sitter space, thermal phase transition, and confinement in gauge theories," Adv. Theor. Math. Phys. 2, 505 (1998) arXiv:hep-th/9803131.

[10] J. X. Lu, S. Roy, Z. Xiao, "Phase transitions and critical behavior of black branes in canonical ensemble," JHEP 1101, 133 (2011). arXiv:1010.2068 [hep-th]]. 
[11] J. X. Lu, S. Roy, Z. Xiao, "Phase structure of black branes in grand canonical ensemble," JHEP 1105, 091(2011) arXiv:1011.5198 [hep-th]].

[12] R. -G. Cai and N. Ohta, "Noncommutative and ordinary superYang-Mills on (D(p 2), D p) bound states," JHEP 0003, 009 (2000) [hep-th/0001213].

[13] W. Taylor, "Adhering zero-branes to six-branes and eight-branes," Nucl. Phys. B 508, 122 (1997) hep-th/9705116.

[14] A. Brandhuber, N. Itzhaki, J. Sonnenschein and S. Yankielowicz, "More on probing branes with branes," Phys. Lett. B 423, 238 (1998) hep-th/9711010].

[15] A. Dhar and G. Mandal, "Probing four-dimensional nonsupersymmetric black holes carrying D0-brane and D6-brane charges," Nucl. Phys. B 531, 256 (1998) hep-th/9803004.

[16] G. W. Gibbons, "Vacuum Polarization and the Spontaneous Loss of Charge by Black Holes," Commun. Math. Phys. 44, 245 (1975).

[17] G. W. Gibbons and D. L. Wiltshire, "Black Holes in Kaluza-Klein Theory," Annals Phys. 167, 201 (1986) [Erratum-ibid. 176, 393 (1987)].

[18] J. X. Lu, S. Roy, Z. Xiao, "The enriched phase structure of black branes in canonical ensemble," Nucl. Phys. B854, 913(2012).

[19] G. T. Horowitz and A. Strominger, "Black strings and P-branes," Nucl. Phys. B 360, 197 (1991).

[20] M. J. Duff and J. X. Lu, "Black and super p-branes in diverse dimensions," Nucl. Phys. B 416, 301 (1994) hep-th/9306052.

[21] M. J. Duff, R. R. Khuri and J. X. Lu, "String solitons," Phys. Rept. 259, 213 (1995) hep-th/9412184.

[22] H. J. Sheinblatt, "Statistical entropy of an extremal black hole with 0-brane and 6-brane charge," Phys. Rev. D 57, 2421 (1998) hep-th/9705054.

[23] G. W. Gibbons and S. W. Hawking, "Action Integrals And Partition Functions In Quantum Gravity," Phys. Rev. D 15, 2752 (1977).

[24] E. Bergshoeff, C. M. Hull and T. Ortin, "Duality in the type II superstring effective action," Nucl. Phys. B 451, 547 (1995) hep-th/9504081]. 
[25] J. C. Breckenridge, G. Michaud and R. C. Myers, "More D-brane bound states," Phys. Rev. D 55, 6438 (1997) [hep-th/9611174].

[26] J. X. Lu and S. Roy, "Non-SUSY p-branes delocalized in two directions, tachyon condensation and T-duality," JHEP 0506, 026 (2005) hep-th/0503007.

[27] J. Polchinski, "Superstring Theory, Vol. 2, Cambridge: Cambridge University Press (1998) 